EPJ Web of Conferences 38, 09002 (2012)

DOI: $10.1051 /$ epjconf/20123809002

(c) Owned by the authors, published by EDP Sciences, 2012

\title{
Description of light charged particle multiplicities in the framework of dinuclear system model
}

\author{
Sh.A. Kalandarov ${ }^{1,2, a}$, G.G. Adamian ${ }^{1}$, and N.V. Antonenko ${ }^{1}$ \\ 1 Joint Institute for Nuclear Research, 141980 Dubna, Russia \\ 2 Institute of Nuclear Physics, 100214 Tashkent, Uzbekistan
}

\begin{abstract}
In the framework of dinuclear system (DNS) model we calculate the light charged particle (LCP) multiplicities produced in fusion and quasifission reactions and their kinetic energy spectra. Calculations indicate that with increasing bombarding energy the ratio of LCP multiplicity from fragments $M_{F F}$ to corresponding LCP multiplicity from compound nucleus $(\mathrm{CN}) M_{C N}$ strongly increases.
\end{abstract}

\section{Introduction}

The phenomenon of light charged particle emission in low energy nuclear reactions is investigated since many years by many authors. The light particles produced in fusionfission and quasifission reactions may be associated with either pre-equilibrium emission or the later evaporation from thermally equilibrated products. In these reactions particle evaporation arises both from the composite nuclear system and from fission-like fragments produced by fusionfission and quasifission processes [1-7]. Coincidence measurements have allowed to use particle emission process to probe the very early stage of nuclear reactions and the individual reaction mechanisms. For example, comparison of measured prescission neutron multiplicities with statistical model predictions reveal the importance of nuclear viscosity in fission process and fission delay time was introduced by Kramers into statistical model. Many authors still try to deduce the fission delay time by fitting the experimental light particle multiplicities by statistical model [8-11]. Consequently, the accuracy of such estimations of fission delay time (nuclear viscosity) is given by statistical models, which has many "not-well-known" parameters allowing to obtain different delay times varying up to 1-3 order of magnitude [12-14]. Authors of Ref. [15,16] recently reported, that the statistical model strongly overestimates proton and alpha particle multiplicities in the ER channel, irrespective of the statistical model input parameters and prescriptions used for the level density and the transmission coefficients. It means that the inclusion of the time delay into statistical model to further suppress the fission process will bring to stronger overestimation of multiplicity data in ${ }^{32} \mathrm{~S}+{ }^{100}$ Mo reaction. Fitting the measured light particle multiplicities by any nuclear decay model does not provide accurate information about fission delay time. Better information can be obtained if one fits simultaneously multiplicity data and cross sections for evaporation residues and fission-like fragments.

The distribution of excitation energy between decay fragments plays important role on particle emission process. Such information can be obtained by measuring the

\footnotetext{
a e-mail: shuhrat@theor.jinr.ru
}

LCP multiplicities in coincidence with decay fragments, since the secondary emission of particles depends on available excitation energy of primary decay fragments. Recently, it was reported that the fragments with $Z>10$ emits LCPs, while the fragments with $Z<10$ do not emit LCPs in the ${ }^{78,82} \mathrm{Kr}+{ }^{40} \mathrm{Ca}$ reaction at the bombarding energy 5.5 MeV/nucleon $[17,18]$. The secondary emission of LCPs from primary decay fragments affects the odd-even staggering effect in charge distributions of final reaction products. So, the theoretical investigations of LCP emission process allows to interpret the experimental results and play an important role in understanding important aspects of the fragmentation process as the sharing of the excitation energies between both partners, odd-even staggering in charge distributions and viscosity phenomenon.

Recently, we developed a model [19] in the framework of DNS concept [20] to calculate the probabilities of all decay channels (light particle evaporation, complex fragment emission, fission and quasifission) in a unique way. The model has been successfully applied for the description of the charge and mass distributions of final decay products, and the cross sections for evaporation residues and fissionlike fragments [21-23]. Here we investigate the process of $\mathrm{LCP}$ emission from $\mathrm{CN}$ and fission-like fragments in fusion and quasifission reactions. We show that the kinetic energy spectra of alpha particles emitted in forward angles from accelerated decay fragments and from direct processes may strongly overlap.

\section{Model}

The DNS model $[19,20]$ describes the evolution of interacting nuclei along two degrees of freedom: the relative distance $R$ coordinate between the center of two nuclei and the charge/mass-asymmetry coordinate, which are defined here by the charge $Z$ and mass $A$ of the light partner of the DNS. After the dissipation of kinetic energy and angular momentum of the relative motion, the DNS is trapped into the pocket of the interaction potential. After the capture stage, there are nucleon drift and nucleon diffusion between the nuclei which constitute the DNS. If the quasifis- 
sion barriers of DNS configurations are enough high compare to neutron binding energy of $\mathrm{CN}$, then the lifetime of such system is enough long to reach a statistical equilibrium in the mass-asymmetry coordinate. In this case the formation probability $P_{Z, A}$ of each DNS or CN configuration depends on the potential energy $U\left(R_{m}, Z, A, J\right)$, calculated with respect to the potential energy of the rotating $\mathrm{CN}$, where $R_{m}$ corresponds to the minima of interaction potential. After the formation of excited $\mathrm{CN}$ and DNS configurations, DNS can decay with a probability $P_{Z, A}^{R}$ in $R$ coordinate if the local excitation energy of the DNS is high enough to overcome the quasifission barrier.

So, the cross section of the binary decay is calculated as follows [19]

$$
\sigma_{Z, A}\left(E_{\text {c.m. }}\right)=\sum_{J=0}^{J_{\max }} \sigma_{c a p}\left(E_{\text {c.m. }}, J\right) W_{Z, A}\left(E_{C N}^{*}, J\right),
$$

where $\sigma_{c a p}$ is the partial capture cross section which defines the transition of the colliding nuclei over the Coulomb barrier and the formation of the initial DNS when the kinetic energy and angular momentum $J$ of the relative motion are transformed into the excitation energy and angular momentum of the DNS. The transition probability is calculated using the Hill-Wheeler formula. $W_{Z, A}\left(E_{C N}^{*}, J\right)$ is the formation-decay probability of the $\mathrm{CN}$ and DNS configurations, which is calculated as the product of corresponding configuration probability and the decay probability:

$$
W_{Z, A}\left(E_{C N}^{*}, J\right)=\frac{P_{Z, A} P_{Z, A}^{R}}{\sum_{Z^{\prime}, A^{\prime}} P_{Z^{\prime}, A^{\prime}} P_{Z^{\prime}, A^{\prime}}^{R}},
$$

where the indexes $Z^{\prime}$ and $A^{\prime}$ goes over all possible channels from the neutron evaporation to the symmetric splitting. The probability $P_{Z, A}$ is the equilibrium limit of the master equation (see [19] for details) given by

$$
P_{Z, A}\left(E_{C N}^{*}, J\right) \sim \exp \left[-U\left(R_{m}, Z, A, J\right) / T_{C N}(J)\right] .
$$

Here, the $n, p, d$ and $t$-evaporation channels are taken into consideration with $U\left(R_{m}, Z, A, J\right)=0$. The quasifission barrier $B_{R}^{q f}$, calculated as the difference between the bottom of the inner pocket and the top of the external barrier, prevents the decay of the DNS along the $R$-degree of freedom with the weight $P_{Z, A}^{R}$ given as

$$
P_{Z, A}^{R} \sim \exp \left[-B_{R}^{q f}(Z, A, J) / T_{Z, A}(J)\right] .
$$

In Eqs. (3) and (4) $T_{C N}(J)$ and $T_{Z, A}(J)$ are the temperatures of the $\mathrm{CN}$ and the DNS, respectively. For the emission of particles with $Z<2, T_{Z, A}(J)=T_{C N}(J)$ and $B_{R}^{q f}(Z, A, J)$ is equal to the particle binding energy plus the value of the Coulomb barrier at $Z \neq 0$. The Fermi-gas model is employed to compute the temperature, with a level-density parameter $a=0.114 A+0.162 A^{2 / 3}$.

In the calculations, we use the formulas (1) and (2) to treat the sequential statistical decay (the evaporation of light particles and/or the binary decay) of the hot $\mathrm{CN}$ and DNS. The generation of whole cascade of decay channels is performed by the Monte Carlo method. We continue to trace the decay processes until all fragments become cold (the excitation energy of fragments is smaller than its neutron emission threshold). The number $n$ of generation of the events in the Monte Carlo technique was chosen according to the smallest decay probability which is $\sim 1 / n$.
Table 1. Excitation energy at $J=0$, maximal kinematical angular momentum, critical angular momentum and capture cross sections for the reactions at given bombarding energies. Energy units are in $\mathrm{MeV}$.

\begin{tabular}{|c|c|c|c|c|c|c|}
\hline Reaction & $E_{\text {lab }}$ & $E_{C N}{ }^{*}$ & $J_{\text {max }}^{\text {kin }}$ & $J_{c r}$ & $J_{0}$ & $\sigma_{c a p}, \mathrm{mb}$ \\
\hline${ }^{16} \mathrm{O}+{ }^{116} \mathrm{Sn}$ & 129.6 & 100 & 70 & 54 & 59 & 1229 \\
${ }^{16} \mathrm{O}+{ }^{116} \mathrm{Sn}$ & 192 & 155 & 96 & 54 & 59 & 830 \\
${ }^{32} \mathrm{~S}+{ }^{100} \mathrm{Mo}$ & 200 & 122 & 98 & 71 & 59 & 918 \\
${ }^{32} \mathrm{~S}+{ }^{100} \mathrm{Mo}$ & 320 & 212 & 149 & 71 & 59 & 574 \\
${ }^{32} \mathrm{~S}+{ }^{109} \mathrm{Ag}$ & 180 & 94 & 83 & 73 & 60 & 1036 \\
${ }^{121} \mathrm{Sb}+{ }^{27} \mathrm{Al}$ & 905 & 134.6 & 105 & 71 & 66 & 925 \\
${ }^{121} \mathrm{Sb}+{ }^{27} \mathrm{Al}$ & 1030 & 157.34 & 118 & 71 & 66 & 813 \\
${ }^{40} \mathrm{Ar}+{ }^{n a t} \mathrm{Ag}$ & 247 & 128 & 118 & 82 & 63 & 857 \\
${ }^{40} \mathrm{Ar}+{ }^{n a t} \mathrm{Ag}$ & 337 & 193 & 160 & 82 & 63 & 628 \\
${ }^{40} \mathrm{Ar}+{ }^{116} \mathrm{Sn}$ & 271 & 139 & 132 & 82 & 64 & 748 \\
${ }^{40} \mathrm{Ar}+{ }^{164} \mathrm{Dy}$ & 340 & 190.6 & 175 & 81 & 69 & 498 \\
\hline
\end{tabular}

Table 2. Lab. energy $E_{\text {lab }}\left(\mathrm{MeV} /\right.$ nucleon) corresponding to $J_{0}$ and $J_{c r}$.

\begin{tabular}{|c|c|c|c|c|}
\hline \multirow{2}{*}{ Reactions } & \multicolumn{2}{|c|}{${ }^{40} \mathrm{Ar}+{ }^{107} \mathrm{Ag}$} & \multicolumn{2}{c|}{${ }^{32} \mathrm{~S}+{ }^{100} \mathrm{Mo}$} \\
\cline { 2 - 5 } & $J_{0}$ & $J_{c r}$ & $J_{0}$ & $J_{c r}$ \\
\hline \multirow{3}{*}{$E_{\text {lab }}$} & 63 & 82 & 59 & 71 \\
& 4.3 & 4.9 & 4.4 & 4.85 \\
\hline
\end{tabular}

\section{Results}

In Table 1 we show the general quantities for the reactions considered in this paper. The maximal angular momentum which contribute to the capture cross section is determined by the minimal value of $J_{\max }^{k i n}, J_{c r}$ [19]. Note, that in our model the maximal angular momentum is not a fitting parameter but it is determined from kinematic conditions and from the existence of the pocket in nucleus-nucleus interaction potential.

$J_{0}$ is the angular momentum at which the potential energy of DNS configuration calculated relatively to $\mathrm{CN}$ energy becomes zero. At this value of angular momentum the probability of finding the system in $\mathrm{CN}$ and DNS configurations becomes close and the mass/charge distributions of decay products begin to transfer from U-shaped form to bell-shaped one. At higher angular momentums, the symmetric DNS configurations becomes energetically more favorable than $\mathrm{CN}$ configuration. In this way, the main source of emitted LCPs change from $\mathrm{CN}$ at small angular momentums to fission-like fragments at high angular momentums. In Table 2 it is shown the bombarding energies at which the maximal angular momentum of the reaction correspond to $J_{0}$ and $J_{c r}$ for a given reactions. It is very interesting to measure the kinetic energy spectra of LCPs at bombarding energies which correspond to the angular momentums $J_{\text {max }}<J_{0}, J_{\text {max }} \approx J_{0}$ and $J_{\text {max }} \approx J_{c r}$ for observing the change of the form of energy spectra due to the change of main emitting source.

In the Tables 3 and 4 we present the comparison of calculated and experimental total cross sections for evaporation residues, fission-like fragments and LCP multiplicities. The overall agreement of calculated results is rather good, although for some systems the disagreement is almost factor of 2-3. For example, for ${ }^{40} \mathrm{Ar}+{ }^{\text {nat }} \mathrm{Ag}$ system at given bombarding energies, our model underestimate the evaporation residues cross sections and reproduce very 
Table 3. Comparison of the calculated and experimental total cross sections for evaporation residues $\sigma_{E R}$ and fission-like fragments $\sigma_{F F}$ (in $\mathrm{mb}$ ). Experimental data are taken from indicated references and corresponding bombarding energies are listed in Table 1.

\begin{tabular}{|c|c|c|c|c|c|}
\hline \multirow{2}{*}{ Reaction } & Ref. & \multicolumn{2}{|c|}{ Calc. } & \multicolumn{2}{|c|}{ Exp. } \\
\cline { 3 - 6 } & & $\sigma_{E R}$ & $\sigma_{F F}$ & $\sigma_{E R}$ & $\sigma_{F F}$ \\
\hline${ }^{16} \mathrm{O}+{ }^{116} \mathrm{Sn}$ & & 1176 & 53 & & \\
${ }^{16} \mathrm{O}+{ }^{116} \mathrm{Sn}$ & & 703 & 127 & & \\
${ }^{32} \mathrm{~S}+{ }^{100} \mathrm{Mo}$ & {$[16]$} & 620 & 298 & $828 \pm 50$ & $130 \pm 13$ \\
${ }^{32} \mathrm{~S}+{ }^{100} \mathrm{Mo}$ & & 258 & 316 & & \\
${ }^{32} \mathrm{~S}+{ }^{109} \mathrm{Ag}$ & & 604 & 432 & & \\
${ }^{121} \mathrm{Sb}+{ }^{27} \mathrm{Al}$ & {$[1]$} & 528 & 397 & $752 \pm 160$ & $280 \pm 60$ \\
${ }^{121} \mathrm{Sb}+{ }^{27} \mathrm{Al}$ & {$[1]$} & 433 & 380 & $690 \pm 130$ & $395 \pm 100$ \\
${ }^{40} \mathrm{Ar}+{ }^{n a t} \mathrm{Ag}$ & {$[3]$} & 335 & 522 & $620 \pm 80$ & $550 \pm 150$ \\
${ }^{40} \mathrm{Ar}+{ }^{n a t} \mathrm{Ag}$ & {$[3]$} & 192 & 436 & $455 \pm 50$ & $520 \pm 150$ \\
${ }^{40} \mathrm{Ar}+{ }^{116} \mathrm{Sn}$ & {$[5]$} & 219 & 529 & $335 \pm 28$ & $503 \pm 74$ \\
${ }^{40} \mathrm{Ar}+{ }^{164} \mathrm{Dy}$ & {$[5]$} & 30 & 480 & $31 \pm 6$ & $1194 \pm 207$ \\
\hline
\end{tabular}

well the cross sections for fission-like fragments, while for a system ${ }^{40} \mathrm{Ar}+{ }^{164} \mathrm{Dy}$ this trend is opposite. The LCPs multiplicities should have the same trend for this reactions, since the multiplicities and corresponding total cross sections are correlated with each other. For ${ }^{40} \mathrm{Ar}+{ }^{n a t} \mathrm{Ag}$ system, alpha particle multiplicities repeat the same tendency as for cross sections, but calculated multiplicities for protons are higher then experimental values. Almost in all cases calculated LCPs multiplicities from fission-like fragments overestimate the experimental values. Since the experimental multiplicities of LCPs are obtained with the procedure of extracting evaporation and direct components from the whole particle energy spectra, the calculation of kinetic energy spectra of LCPs in the framework of our model is very demanding to resolve the origin of such discrepancies. The analysis of kinetic energy spectra of emitted LCPs in the framework of our model is our future aim.

In figure 1 we present the dependence of LCP multiplicities for ${ }^{40} \mathrm{Ar}+{ }^{\text {nat }} \mathrm{Ag}$ system from bombarding energy. With increasing bombarding energy, the proton and alpha particle multiplicities from fission-like fragments strongly increases. This is due to the increase of angular momentum, since with increasing angular momentum, probability of finding the system in symmetric DNS configurations strongly increases and quasifission products become the main source of emitted LCPs.

\section{Conclusion}

In the framework of DNS model we investigate the emission of LCPs from excited $\mathrm{CN}$ and fission-like fragments in fusion and quasifission reactions. The calculated total cross sections for evaporation residues, fission like fragments and LCPs multiplicities are in good agreement with experimental data, although in some cases the disagreement is up to factor of 2-3. Calculation show that the with increasing bombarding energy the ratio of LCP multiplicity from fragments $M_{F F}$ to corresponding LCP multiplicity from compound nucleus $(\mathrm{CN}) M_{C N}$ strongly increases due to the increase of quasifission probability. With increasing bombarding energy, the kinetic energy spectra of emitted LCPs should also change the form and peak positions due

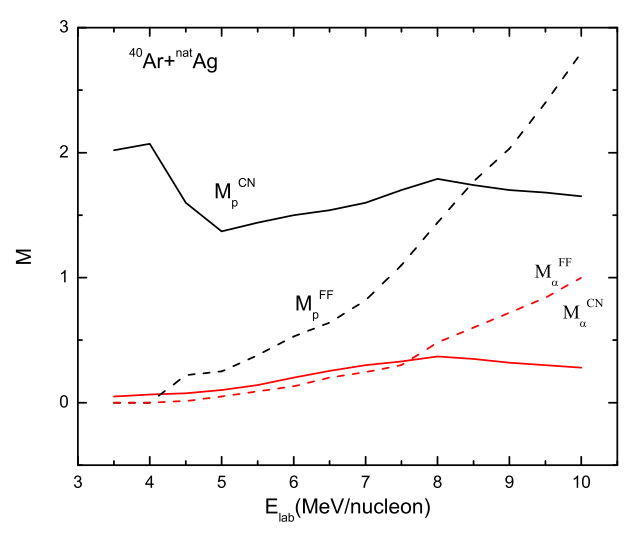

Fig. 1. Bombarding energy dependence of multiplicities of protons and alpha particles from $\mathrm{CN}$ and fission like fragments.

to the change of main emitting source of LCPs.

\section{Acknowledgements}

This work was supported by DFG and RFBR. IN2P3 JINR and Polish-JINR Cooperation programs are gratefully acknowledged.

\section{References}

1. W. E. Parker et al., Nucl. Phys. A 568, 633 (1994)

2. H. C. Britt et al., Phys. Rev. C 13, 1483 (1976)

3. R. Lacey et al., Phys. Rev. C 37, 2540 (1988)

4. A.G. Artukh et al., Z. Phys. A - Atoms and Nuclei 303, $41(1981)$

5. D. Logan et al., Phys. Rev. C 22, 1080 (1980)

6. H. Singh et al., Phys. Rev. C 78, 024609 (2008)

7. J. Cabrera et al., Phys. Rev. C 68, 034613 (2003)

8. K. Ramachandran et al., Phys. Rev. C 73, 064609 (2006)

9. V. A. Rubchenya et al., Phys. Rev. C 58, 1587 (1998)

10. A. Saxena et al., Nucl. Phys. A 730, 299 (2004)

11. R. P. Schmitt et al., Phys. At. Nucl. 66, 1163 (2003)

12. J.P. Lestone, Phys. Rev. C 59, 1549 (1999)

13. D.J. Hofman, B.B. Back, P. Paul, Nucl. Phys. A 599, 23c (1996)

14. H. Ikezoe et al., Phys. Rev. C 49, 968 (1994)

15. A. Di Nitto et al., Eur. Phys. J. A 47, 83 (2011)

16. E. Vardaci et al., Eur. Phys. J. A 43, 127 (2010)

17. G. Ademard et al., Phys. Rev. C 83, 054619 (2011)

18. G. Ademard et al., EPJ Web of Conf. 17, 10005 (2011)

19. Sh.A. Kalandarov, G.G. Adamian, N.V. Antonenko, W. Scheid, Phys. Rev. C 82, 044603 (2010)

20. V.V. Volkov, Izv. AN SSSR, ser. fiz. 50, 1879 (1986); G.G. Adamian, N.V. Antonenko, and W. Scheid, Nucl. Phys. A 618, 176 (1997); G.G. Adamian, N.V. Antonenko, W. Scheid, and V.V. Volkov, Nucl. Phys. A 627, 361 (1997); G.G. Adamian, N.V. Antonenko, W. Scheid, and V.V. Volkov, Nucl. Phys. A 633, 409 (1998) 
Table 4. Comparison of the calculated and experimental multiplicities of protons $M_{p}$ and alpha particles $M_{\alpha}$ from CN and from fissionlike fragments. Experimental data are taken from indicated references.

\begin{tabular}{|c|c|c|c|c|c|c|c|c|c|c|}
\hline Reaction & Ref. & $E_{\text {lab }}, \mathrm{MeV}$ & \multicolumn{2}{c|}{$M_{C N}$ (Calc. $)$} & \multicolumn{2}{c|}{$M_{F F}$ (Calc. } & \multicolumn{2}{c|}{$M_{C N}$ (Exp.) } & \multicolumn{2}{c|}{$M_{F F}$ (Exp.) } \\
\cline { 4 - 10 } & & & $M_{p}$ & $M_{\alpha}$ & $M_{p}$ & $M_{\alpha}$ & $M_{p}$ & $M_{\alpha}$ & $M_{p}$ & $M_{\alpha}$ \\
\hline${ }^{16} \mathrm{O}+{ }^{116} \mathrm{Sn}$ & & 129.6 & 1.66 & 1.03 & 0.01 & 0.005 & - & - & - & - \\
${ }^{16} \mathrm{O}+{ }^{116} \mathrm{Sn}$ & & 192 & 2.3 & 1.26 & 0.169 & 0.08 & - & - & - & - \\
${ }^{32} \mathrm{~S}+{ }^{100} \mathrm{Mo}$ & {$[16]$} & 200 & 0.99 & 0.83 & 0.2 & 0.11 & $0.9 \pm 0.14$ & $0.56 \pm 0.09$ & $0.055 \pm 0.007$ & $0.038 \pm 0.005$ \\
${ }^{32} \mathrm{~S}+{ }^{100} \mathrm{Mo}$ & & 320 & 1.2 & 0.9 & 0.62 & 0.28 & - & - & - & - \\
${ }^{32} \mathrm{~S}+{ }^{109} \mathrm{Ag}$ & {$[15]$} & 180 & 1.42 & 0.4 & 0.33 & 0.065 & $1.3 \pm 0.3$ & $0.6 \pm 0.1$ & - & - \\
${ }^{121} \mathrm{Sb}+{ }^{27} \mathrm{Al}$ & {$[1]$} & 905 & 1.58 & 0.36 & 0.22 & 0.1 & $1.156 \pm 0.26$ & $0.734 \pm 0.17$ & $0.132 \pm 0.02$ & $0.134 \pm 0.02$ \\
${ }^{121} \mathrm{Sb}+{ }^{27} \mathrm{Al}$ & {$[1]$} & 1030 & 1.7 & 0.46 & 0.378 & 0.166 & $1.409 \pm 0.29$ & $1.122 \pm 0.23$ & $0.256 \pm 0.038$ & $0.213 \pm 0.032$ \\
${ }^{40} \mathrm{Ar}+{ }^{n a t} \mathrm{Ag}$ & {$[3]$} & 247 & 1.5 & 0.22 & 0.58 & 0.146 & $1.02 \pm 0.2$ & $0.5 \pm 0.1$ & $0.127 \pm 0.015$ & $0.1 \pm 0.017$ \\
${ }^{40} \mathrm{Ar}+{ }^{n a t} \mathrm{Ag}$ & {$[3]$} & 337 & 1.79 & 0.37 & 1.44 & 0.48 & $2.1 \pm 0.6$ & $1.4 \pm 0.4$ & $0.438 \pm 0.2$ & $0.437 \pm 0.044$ \\
${ }^{40} \mathrm{Ar}+{ }^{116} \mathrm{Sn}$ & {$[5]$} & 271 & 0.9 & 0.285 & 0.56 & 0.246 & 1.28 (total) & $0.74($ total $)$ & & \\
${ }^{40} \mathrm{Ar}+{ }^{164} \mathrm{Dy}$ & {$[5]$} & 340 & 0.23 & 0.15 & 0.6 & 0.25 & 0.4 & 0.14 & 0.69 & 0.8 \\
\hline
\end{tabular}

21. Sh.A. Kalandarov, G.G. Adamian, N.V. Antonenko, W. Scheid, Phys. Rev. C 83, 054611 (2011)

22. Sh.A. Kalandarov, G.G. Adamian, N.V. Antonenko, W. Scheid, J.-P. Wieleczko, Phys. Rev. C 84, 064601 (2011)

23. Sh.A. Kalandarov et al., Phys. Rev. C 84, 054607 (2011) 